\title{
CONSTRUÇÃO E VALIDAÇÃO DE UM INSTRUMENTO PARA AVALIAÇÃO DA REGULAÇÃO EMOCIONAL
}

\author{
José Maurício Haas Bueno4 \\ Doutor em Psicologia, Universidade Federal de Pernambuco.
}

\begin{abstract}
Resumo
Considerando a inexistência de um instrumento para avaliação de habilidades relacionadas à inteligência emocional (IE) no mercado brasileiro e as relações que a regulação de emoções (uma das habilidades relacionadas com a IE) vem mostrando com critérios externos, esse trabalho propõe a construção e investigação das propriedades psicométricas de um instrumento para avaliação da regulação emocional. Para tanto, foram construídas provas baseadas na técnica do Stroop Emocional, utilizando palavras e imagens como estímulos emocionais e a nomeação de cores como tarefa cognitiva. Esse instrumento foi aplicado a uma amostra de 207 participantes adultos, de ambos os sexos. Uma análise fatorial revelou dois fatores que se estruturaram em função do tipo de estímulo emocional (imagens e palavras), porém esses fatores apresentaram um padrão de correlações neutras com traços de personalidade e raciocínio abstrato. Esses resultados foram discutidos à luz de pesquisas anteriores e caminhos foram apontados para a continuidade do desenvolvimento do instrumento.
\end{abstract}

Palavras-chave: regulação de emoções; inteligência emocional; inteligência; personalidade.

\section{CONSTRUCTION AND VALIDATION OF NA INSTRUMENT FOR EMOTIONAL REGULATION ASSESSMENT}

\begin{abstract}
Considering the lack of an instrument for assessing emotional intelligence (EI) related skills in the Brazilian market and the relationships that emotional regulation (one of the skills related to IE) has shown with external criteria, this work proposes the construction and investigation of the psychometric properties of an instrument for emotional regulation assessment. To this end, an instrument was constructed based on Emotional Stroop task, using words and pictures as emotional stimuli and color naming as cognitive task. This instrument was administered to 207 adults, of both sexes. A factor analysis revealed two factors that were structured according to the type of emotional stimuli (pictures and words), but these factors showed a standard of neutral correlations with personality traits and abstract reasoning. These results were discussed in light of previous research and ways were appointed to continuing the development of the instrument.
\end{abstract}

Keywords: emotional regulation; emotional intelligence; intelligence; personality.

${ }^{4} \mathrm{O}$ autor recebeu financiamento do CNPq. 


\title{
CONSTRUCCIÓN Y VALIDACIÓN DE UN INSTRUMENTO PARA EVALUAR LA REGULACIÓN EMOCIONAL
}

\begin{abstract}
Resumen
Considerando la falta de un instrumento para evaluar las habilidades relacionadas con la inteligencia emocional (IE) en el mercado brasileño y de las relaciones que la regulación de las emociones (una de las habilidades relacionadas con la IE) ha demostrado con criterios externos, este trabajo propone la construcción y la investigación de las propiedades psicométricas de un instrumento para evaluar la regulación emocional. Con este fin, fueron construidas pruebas basadas en la técnica de Stroop emocional, utilizando palabras e imágenes como estímulos emocionales y nombres de colores como tarea cognitiva. Este instrumento fue administrado a una muestra de 207 participantes adultos de ambos los sexos. Un análisis factorial reveló dos factores que se estructuraran de acuerdo con el tipo de estímulos emocionales (imágenes y palabras), pero estos factores mostraron un patrón de correlaciones neutras con rasgos de personalidad y el razonamiento abstracto. Estos resultados son discutidos a la luz de las investigaciones anteriores y caminos fueron indicados para continuación del desarrollo del instrumento.

Palabras clave: regulación emocional; inteligencia emocional; inteligencia;
\end{abstract} personalidad.

\section{INTRODUÇÃO}

A inteligência emocional (IE) foi proposta como um tipo de inteligência relacionada ao processamento cognitivo de informações emocionais (Salovey \& Mayer, 1990). Ela foi explicada por meio de um sistema de quarto níveis, organizados de acordo com a complexidade do processo psicológico subjacente a cada um deles, que são os seguintes: (a) percepção de emoções; (b) utilização da emoção para facilitação do pensamento; (c) compreensão emocional e (d) regulação emocional (Mayer \& Salovey, 1997). Posteriormente, foi encontrado que essas habilidades poderiam se agrupar em duas áreas hierarquicamente superiores, denominadas por área experiencial (junção da percepção de emoções com a facilitação do pensamento) e área estratégica (junção da compreensão emocional com a regulação emocional). Finalmente, essas duas áreas se fundiam em apenas um fator capaz de explicar o desempenho geral em inteligência emocional (Mayer, Salovey \& Caruso, 2002).

Dentro desse modelo, o principal instrumento utilizado para avaliação da inteligência emocional tem sido o Mayer-Salovey-Caruso Emotional Intelligence Test (MSCEIT) (Mayer, Salovey \& Caruso, 2002). Nesse instrumento cada uma das quatro habilidades básicas é avaliada por duas provas primárias, de modo que o teste todo é composto por oito tarefas dispostas em 141 itens. Vários estudos atestaram sua validade fatorial, embora com alguma discrepância quanto o número de fatores encontrados (Bueno, 2008; Cobêro, 2004; Dantas, 
2004; Jesus Jr., 2004; Mayer, Caruso \& Salovey, 1999; Mayer, Salovey, Caruso \& Sitarenios, 2003; Palmer, Gignac, Manocha \& Stough, 2005; Zeidner, Matthews \& Roberts, 2001), assim como sua convergência com medidas tradicionais de inteligência (Cobêro, Primi \& Muniz, 2006; Mayer e cols., 1999; Pelletteri, 1999) e independência de traços de personalidade (Bastian, Burns \& Nettelbeck, 2005; Caruso, Mayer \& Salovey, 2002).

Esses estudos foram realizados dentro de uma concepção da IE como uma aptidão, que a compreende como uma habilidade a ser avaliada por meio de instrumentos de desempenho ao invés de instrumentos de autorrelato (ver Mayer, Salovey \& Caruso, 2000). No entanto, a habilidade de regulação emocional, por exemplo, é avaliada no MSCEIT por meio da apresentação de relatos situacionais em que um personagem vivencia uma emoção e o sujeito é solicitado a escolher a alternativa de reação mais apropriada para a situação (Mayer, Salovey \& Caruso, 2002). Nesse caso, o que o instrumento avalia é mais o conhecimento de como regular do que de que a habilidade de regular emoções propriamente dita, aproximando-se mais das características de um instrumento de autorrelato do que de desempenho.

Uma possível alternativa ao procedimento existente seria a utilização de uma prova de Stroop Emocional. Nessa prova, palavras neutras ou carregadas emocionalmente são apresentadas aos sujeitos, que devem indicar a cor em que as palavras estão escritas. Geralmente, quando se toma o tempo decorrido entre a apresentação da palavra e a escolha da cor (tempo de reação), observa-se que as palavras emocionalmente carregadas produzem um tempo de reação maior do que as emocionalmente neutras. Isto parece ser verdade, especialmente para pessoas que têm dificuldade em gerenciar suas emoções, como as ansiosas (Ashley \& Swick, 2009; Fava, Kristensen, Melo \& Araujo, 2009), depressivas (Gotlib \& McCann, 1984) ou abusadoras sexuais (Smith \& Waterman, 2004), por exemplo. Nesse tipo de prova, o controle emocional tem que ser efetivamente utilizado para diminuir o tempo de reação a palavras emocionalmente carregadas e fica clara a existência de diferenças individuais nesse tempo em função da (des)organização mental.

É possível que essas diferenças individuais também sejam observadas em uma tarefa não verbal em que os estímulos distratores fossem imagens, selecionadas da coleção do International Affective Picture System - IAPS, de acordo com os índices de valência e arousal (Bradley \& Lang, 2007), e a tarefa 
fosse algo como localizar e nomear a cor de um círculo padronizado sobre cada imagem. A expectativa seria a de que o tempo de reação variasse em função da maior ou menor neutralidade emocional do estímulo de fundo e da própria capacidade de controle emocional do indivíduo.

$O$ investimento no desenvolvimento de tal instrumento pode ser interessante em razão das relações que a regulação emocional tem apresentado com critérios externos (validade com base na relação com outras variáveis). Por exemplo, Freitas e Noronha (2006) verificaram que a regulação emocional foi a principal habilidade da IE a se relacionar com o desempenho em uma tarefa de psicodiagnóstico em estudantes de Psicologia, enquanto Cobêro e colaboradores (2006) encontraram evidências de que a regulação emocional apresenta validade incremental para desempenho no trabalho em relação a medidas tradicionais de inteligência. Infelizmente, não há atualmente no mercado brasileiro um instrumento para avaliação dessa habilidade, o que pode representar uma deficiência tanto nas diversas áreas de atuação do psicólogo quanto para a realização de pesquisas nessa área.

Por esses motivos, a proposta deste trabalho foi desenvolver um instrumento para avaliação da regulação emocional por desempenho e avaliar suas propriedades psicométricas. Mais especificamente, esperava-se que: (a) as diferenças entre os tempos dos blocos com estímulos emocionais e o bloco com estímulos neutros (tempos de latência) fossem positivas e estatisticamente significativas; (b) que essas medidas formassem uma estrutura unidimensional relacionada à regulação de emoções, ou, no máximo, duas dimensões relacionadas ao tipo de estímulo; (c) que os fatores encontrados apresentassem consistência interna superior a 0,7 ; (d) que houvesse correlação positiva e estatisticamente significativa entre os fatores de regulação emocional, caso fosse encontrado mais de um; (e) que as provas de regulação emocional se correlacionassem moderadamente (acima de 0,25 ) com uma medida tradicional de inteligência, o raciocínio abstrato e que apresentasse um padrão de correlações baixas (menores que 0,25 ) com traços de personalidade.

\section{MÉTODO}

\section{Participantes}

Foram participantes da pesquisa 207 sujeitos, com média de 30,3 anos de idade $(D P=12,7)$, sendo $56,5 \%$ do sexo feminino. A maioria dos participantes era 
solteiro $(62,0 \%)$ e exercia ou estava engajado numa profissão ligada à área de ciências humanas $(43,0 \%), 62,8 \%$ viveu nos últimos dez anos predominantemente em cidades de grande porte (com mais de 800.000 habitantes), 62,3\% apresentava curso superior completo ou incompleto e 43,5\% apresentava renda familiar superior a 5 salários mínimos.

\section{Instrumentos}

Para caracterização da amostra de participantes desta pesquisa, foi usado um Questionário de Identificação Pessoal, que coletou informações sobre idade, sexo, escolaridade, estado civil, naturalidade e faixa de renda familiar. E, para atingir os objetivos propostos nesta pesquisa, foram aplicados três instrumentos psicológicos, cujas características são descritas a seguir.

O Teste de Regulação Emocional (TRE) foi construído com base na técnica do Stroop Emocional, em que uma tarefa cognitiva (nomear cores) concorre com a interferência de conteúdos emocionais. No caso do TRE, esse princípio foi aplicado a estímulos verbais e a não-verbais. Os estímulos não-verbais eram imagens selecionadas do International Affective Picture System (IAPS) (Bradley \& Lang, 2007; Lang, Bradley \& Cuthbert, 2005; Lasaitis, Ribeiro, Freire \& Bueno, 2008; Ribeiro, Pompéia \& Bueno, 2004), sobre as quais foram adicionados círculos coloridos (um por imagem), de aproximadamente 1,5 cm de diâmetro, na porção central horizontal da imagem (no centro, à direita ou à esquerda). Os estímulos verbais eram palavras, em português, selecionadas das Normas Brasileiras para o Affective Norms for English Words (Kristensen, Gomes, Justo \& Vieira, 2011), que eram grafadas em diferentes cores. Tanto os pontos sobre as imagens quanto as palavras apareciam equitativamente em quatro cores: amarelo, vermelho, verde e azul. Em ambos os tipos de estímulo havia quinze itens de valência neutra, quinze com valência negativa associadas à emoção de ansiedade, quinze com valência negativa associadas à emoção de tristeza e quinze com valência positiva. Antes desses estímulos, contudo, havia uma série de 15 estímulos, extraídos do banco de imagens do Microsoft Office, para familiarização com a tarefa de nomeação de cores. Foram cronometrados manualmente os tempos decorrentes para a nomeação das cores de todos os 15 estímulos de cada série, de modo que cada sujeito obteve oito cronometragens: imagens neutras, imagens negativas de ansiedade, imagens positivas, imagens negativas de tristeza, palavras neutras, palavras negativas de ansiedade, palavras positivas e palavras negativas de tristeza. Posteriormente, os tempos 
para nomeação de cores em estímulos neutros foram subtraídos dos tempos para nomeação de cores em estímulos emocionais de modo que cada participante obteve três escores (ansiedade, emoção positiva e tristeza) para cada tipo de estímulo (Imagens - I e Palavras - P), perfazendo seis escores no total: I_ansiedade, I_positiva, I_tristeza, P_ansiedade, P_positiva e P_tristeza. Dessa forma, escores positivos indicavam que os estímulos emocionais dificultaram a realização da tarefa e os escores negativos indicavam que os estímulos emocionais facilitaram a realização da tarefa, em relação ao tempo para nomeação de estímulos neutros.

Os testes foram aplicados com auxílio de um arquivo construído em Power Point, que apresentava as instruções e conduzia o participante ao longo do teste. Um tempo de aproximadamente 15 segundos era dado entre a aplicação de um bloco de 15 estímulos e o seguinte. Os tempos eram cronometrados e anotados pelo aplicador.

A Prova de Raciocínio Abstrato (RA), Forma B, da Bateria de Provas de Raciocínio (BPR-5) apresenta 25 itens, em formato de analogias, do tipo $A \rightarrow B / C \rightarrow$ ? (cinco alternativas), para avaliação do raciocínio indutivo. Associa-se, portanto, principalmente à inteligência fluida (Gf), definida como capacidade de raciocinar em situações novas, criar conceitos e compreender implicações. Os índices de precisão dessa prova, informados no manual do instrumento, foram de 0,72 e 0,85, para o método das metades e o coeficiente alfa, respectivamente. Como evidência de validade, o manual informa que a prova saturou em um fator único juntamente com provas de raciocínio numérico, espacial, verbal e mecânico, atestando sua validade para avaliação do fator g. O teste foi aplicado conforme as recomendações constantes no manual do instrumento, com o tempo máximo de 12 minutos para sua execução e o cálculo da pontuação total pela soma de respostas corretas dadas aos 25 itens (Primi \& Almeida, 2000).

A Bateria Fatorial de Personalidade (BFP) é um instrumento para avaliação dos Cinco Grandes Fatores de Personalidade: Neuroticismo (cujos indicadores são os traços de vulnerabilidade, instabilidade emocional, passividade/falta de energia e depressão), Extroversão (cujos indicadores são os traços de comunicação, altivez, dinamismo e interações sociais), Socialização (cujos indicadores são os traços de Amabilidade, Pró-Sociabilidade, Confiança nas Pessoas), Realização (cujos indicadores são os traços de Competência, Ponderação/Prudência, Empenho/Comprometimento) e Abertura à Experiência 
(cujos indicadores são os traços de abertura à ideias, liberalismo e busca por novidades). A bateria é composta por 126 afirmações, que os sujeitos são convidados a ler e a julgar o quanto se aplicam ao seu próprio caso, utilizando para isso uma escala tipo Likert de sete pontos, em que 1 significa "descreve-me muito mal" e 7 significa "descreve-me muito bem". Estudos evidenciaram bons índices de fidedignidade, além de validade fatorial e com variáveis externas. 0 instrumento foi aplicado e avaliado conforme instruções constantes no manual (Nunes, Hutz \& Nunes, 2010).

Procedimentos

Antes de ser executado, o projeto desta pesquisa foi submetido ao escrutínio de um Comitê de Ética em Pesquisa, que emitiu parecer favorável à sua realização. Assim, os sujeitos foram contatados em salas de aula e em suas casas, por aplicadores que estudaram e foram submetidos à aplicação dos instrumentos antes de aplicarem em outras pessoas. Os participantes foram esclarecidos quanto aos objetivos, procedimentos e formas de contato com os pesquisadores e os que concordaram com os termos de participação, assinaram um Termo de Consentimento Livre e Esclarecido, conforme as recomendações éticas para pesquisas com seres humanos.

O Teste de Regulação de Emoções foi aplicado sempre individualmente juntamente com o questionário de informações pessoais. Já a Prova de Raciocínio Abstrato e a Bateria Fatorial de Personalidade foram aplicadas coletivamente em alguns casos e individualmente em outros, dependendo da forma como cada participante foi abordado. Em todos os casos, garantiu-se um ambiente tranquilo para a realização das tarefas propostas. Os dados coletados foram digitados em planilha eletrônica para a realização das análises estatísticas.

\section{RESULTADOS}

O primeiro passo na realização das análises estatísticas foi o cálculo das pontuações nos instrumentos, conforme descrito anteriormente. Em seguida, calculou-se os tempos de latência, pela subtração do tempo para nomeação das cores do bloco de estímulos neutros dos tempos para nomeação de cores dos blocos de estímulos emocionais. A Tabela 1 apresenta tanto as estatísticas descritivas de cada uma dessas variáveis como os testes t para verificação das diferenças entre médias. 
Tabela 1.

Estatísticas descritivas e testes $t$.

\begin{tabular}{|c|c|c|c|c|c|c|c|c|c|}
\hline & \multicolumn{6}{|c|}{ Tempos Totais } & \multicolumn{2}{|c|}{ Latência } \\
\hline & & Média & $\mathrm{N}$ & $\mathrm{DP}$ & $t$ & gl & $p$ & Média & $\mathrm{DP}$ \\
\hline Par & $\begin{array}{l}\text { I_Ans } \\
\text { I_Neu }\end{array}$ & $\begin{array}{l}13,39 \\
12,81\end{array}$ & $\begin{array}{l}206 \\
206 \\
\end{array}$ & $\begin{array}{l}2,85 \\
2,61\end{array}$ & 4,32 & 205 & 0,000 & 0,57 & 1,90 \\
\hline Par 2 & $\begin{array}{l}\text { I_Pos } \\
\text { I_Neu }\end{array}$ & $\begin{array}{l}12,85 \\
12,82 \\
\end{array}$ & $\begin{array}{l}206 \\
206 \\
\end{array}$ & $\begin{array}{l}2,41 \\
2,61 \\
\end{array}$ & 0,24 & 205 & 0,8 & 0,03 & 1,85 \\
\hline Par 3 & $\begin{array}{l}\text { I_Tris } \\
\text { I_Neu }\end{array}$ & $\begin{array}{l}13,43 \\
12,81\end{array}$ & $\begin{array}{l}206 \\
206\end{array}$ & $\begin{array}{l}2,97 \\
2,60\end{array}$ & 8 & 05 & 0,000 & 0,62 & 2,25 \\
\hline Par 4 & $\begin{array}{l}\text { P_Ans } \\
\text { P_Neu }\end{array}$ & $\begin{array}{l}14,44 \\
14,30 \\
\end{array}$ & $\begin{array}{l}207 \\
207 \\
\end{array}$ & $\begin{array}{l}3,00 \\
2,84\end{array}$ & 1,07 & 206 & & 0,14 & 1,83 \\
\hline Par 5 & $\begin{array}{l}\text { P_Pos } \\
\text { P_Neu }\end{array}$ & $\begin{array}{l}13,81 \\
14,30 \\
\end{array}$ & $\begin{array}{l}207 \\
207 \\
\end{array}$ & $\begin{array}{l}2,79 \\
2,84 \\
\end{array}$ & $-3,87$ & 206 & 0,000 & $-0,49$ & 1,82 \\
\hline Par 6 & $\begin{array}{l}\text { P_Tris } \\
\text { P_Neu }\end{array}$ & $\begin{array}{l}14,09 \\
14,30\end{array}$ & $\begin{array}{l}207 \\
207\end{array}$ & $\begin{array}{l}2,79 \\
2,84\end{array}$ & $-1,63$ & 206 & 0,105 & $-0,21$ & 1,90 \\
\hline
\end{tabular}

Nota: I - imagens; P - palavras

Esperava-se que os tempos de latência fossem positivos e as diferenças estatisticamente significativas. No entanto, os dados indicam comportamentos diferentes dos sujeitos perante imagens e palavras. Em relação às imagens o comportamento foi tal como esperado para os blocos de emoções negativas (ansiedade e tristeza), pois, em média, os sujeitos apresentaram um tempo de reação significativamente maior para esses blocos em comparação ao bloco de palavras neutras, resultando em valores positivos de latência. Contudo, o tempo de reação para o bloco de imagens associadas a emoções positivas foi pouco superior ao de nomeação de cores sobre imagens neutras, resultando numa diferença não significativa muito próxima de zero.

Inesperadamente, quando o estímulo mudou para palavras, a situação foi bastante diferente. Apenas o bloco de palavras associadas a ansiedade apresentou tempo médio de latência positivo, indicando tempo de reação maior a essas palavras do que às neutras, porém com diferença não significativa. Diferentemente, os blocos de palavras associadas a emoções positivas e à tristeza apresentaram tempo de reação menor do que ao bloco de palavras neutras (tempo de latência negativo). No entanto, como se pode observar, a única diferença estatisticamente significativa foi entre os tempos para nomeação de cores em palavras positivas e neutras.

Esse comportamento diferenciado entre os tempos de reação dos diferentes tipos de estímulo foi confirmado por uma análise fatorial cujos fatores extraídos 
por fatoração dos eixos principais e rotação varimax revelou uma estrutura bifatorial, capaz de explicar 55,6\% da variância total. As cargas fatoriais e os coeficientes de consistência de cada fator são apresentados na Tabela 2.

Tabela 2.

Cargas fatoriais e coeficientes alfa de Cronbach

\begin{tabular}{ccc}
\hline & \multicolumn{2}{c}{ Fatores } \\
\cline { 2 - 3 } & \multicolumn{1}{c}{1} & 2 \\
\hline P_pos & 0,780 & \\
P_ans & 0,763 & \\
P_tris & 0,685 & \\
I_pos & & 0,801 \\
I_tris & & 0,765 \\
I_ans & & 0,655 \\
\hline
\end{tabular}

Coeficiente Alfa de Cronbach $0,789 \quad 0,780$

Conforme esperado, foram obtidos dois fatores organizados pelo tipo de estímulo emocional apresentado: palavras e imagens. Em ambos os casos os coeficientes de consistência interna também corresponderam às expectativas, ficando acima de 0,7 , indicando que as diferenças individuais nos fatores podem ser atribuídas ao que os fatores medem e não a erros de medida. O problema que se apresentou aqui foi saber o que exatamente os fatores medem. Para tentar responder a essa pergunta, foram calculadas as pontuações dos sujeitos nos fatores obtidos e verificadas as correlações entre eles e deles com outras variáveis (inteligência e personalidade).

A nomeação de cores em palavras emocionais variou de $-6,49$ a 4,08, com média igual a -0,19 e desvio padrão igual a 1,55, enquanto a nomeação de cores em imagens emocionais variou de $-5,41$ a 5,13, com média igual a 0,40 e desvio padrão igual a 1,69. Esses dados refletem a observação inicial de que as imagens haviam produzido maior tempo de latência do que as palavras em relação aos estímulos neutros. Ao contrário do esperado, a correlação entre os fatores foi de 0,071, não-significativa. As correlações desses fatores com a medida de inteligência (raciocínio abstrato) e com traços de personalidade são apresentadas na Tabela 3. 
Tabela 3.

Coeficientes de correlação de Pearson

\begin{tabular}{lcc}
\hline & Palavras & Imagens \\
\hline Raciocínio Abstrato & 0,009 & 0,035 \\
Vulnerabilidade (N1) & $-0,068$ & 0,040 \\
Instabilidade Emocional (N2) & 0,005 & 0,041 \\
Passividade / Falta de Energia (N3) & 0,017 & 0,007 \\
Depressão (N4) & $-0,151^{*}$ & 0,046 \\
Neuroticismo (N) & $-0,056$ & 0,045 \\
Comunicação (E1) & $-0,054$ & 0,011 \\
Altivez (E2) & $-0,034$ & 0,016 \\
Dinamismo (E3) & 0,022 & $-0,027$ \\
Interações Sociais (E4) & $-0,037$ & 0,022 \\
Extroversão (E) & $-0,035$ & 0,008 \\
Amabilidade (S1) & $-0,116$ & $-0,062$ \\
Pró-Sociabilidade (S2) & $-0,011$ & $-0,035$ \\
Confiança nas Pessoas (S3) & 0,091 & $-0,022$ \\
Socialização (S) & $-0,009$ & $-0,050$ \\
Competência (R1) & $-0,027$ & 0,123 \\
Ponderação / Prudência (R2) & 0,016 & $-0,038$ \\
Empenho / Comprometimento (R3) & $-0,075$ & 0,088 \\
Realização (R) & $-0,010$ & 0,082 \\
Abertura a ideias (A1) & 0,027 & 0,066 \\
Liberalismo (A2) & $0,176^{*}$ & 0,131 \\
Busca por Novidades (A3) & 0,037 & 0,063 \\
Abertura (A) & 0,111 & 0,123 \\
\hline Correlacos estatisticamente
\end{tabular}

* Correlações estatisticamente significativas ao nível 0,05 (bicaudal).

Como se pode observar, as correlações foram todas baixas e apenas duas significativas ao nível de 0,05 , ambas relacionadas à tarefa com palavras. No primeiro caso, o traço de depressão ficou inversamente associado ao tempo para nomeação de palavras emocionais, o que não corresponde ao que vem sendo encontrado na literatura sobre o assunto. No segundo caso, o traço de liberalismo associou-se positivamente ao tempo para nomeação de palavras emocionais. Neste caso, embora não tenha sido encontrada informações anteriores sobre esse tipo de relação entre as variáveis, elas fazem sentido, pois uma pessoa mais aberta aos sentimentos, tende a se impressionar e focar a atenção em eventos carregados de afeto. No entanto, ambas as correlações 
foram muito baixas e necessitariam ser confirmadas por outros estudos para serem tomadas como consistentes.

\section{DISCUSSÃO}

O objetivo geral deste trabalho foi construir e avaliar as propriedades psicométricas de um instrumento baseado na técnica do Stroop Emocional para avaliação da capacidade de regular emoções. Em relação aos objetivos específicos, o instrumento apresentou resultados irregulares, que ora confirmavam as expectativas, ora as contradiziam. Assim, por um lado, o instrumento apresentou estrutura bifatorial relacionada ao tipo de estímulo (imagens ou palavras) com bons índices de consistência interna; por outro, esses fatores não se correlacionaram entre si, nem com traços de personalidade e inteligência.

Algumas hipóteses explicativas podem ser levantadas para explicar esse fato. A primeira delas se refere ao funcionamento da tarefa de Stroop Emocional em amostras não clínicas de participantes. Por exemplo, Cisler e colaboradores (2011) encontraram diferenças entre os tempos de reação a palavras neutras e ameaçadoras em grupos de pacientes com transtorno do estresse pós-traumático (TEPT) e em um grupo de pacientes expostos a trauma, mas sem TEPT. No entanto, a mesma diferença não foi observada em um grupo controle de participantes, sem qualquer tipo de exposição a trauma. Nesse sentido, os participantes da presente pesquisa se assemelham ao do último grupo, já que não houve nenhum critério de inclusão ou exclusão de participantes da pesquisa.

De fato, os participantes da presente pesquisa não apresentaram diferenças significativas entre os tempos para nomeação de cores de palavras associadas a emoções negativas (ansiedade e tristeza) e neutras. No entanto, a utilização de imagens no lugar de palavras faz com que o tempo para nomeação de cores seja significativamente maior para palavras emocionalmente negativas (ansiedade e tristeza) em relação às neutras, mostrando clara interferência do conteúdo emocional sobre a tarefa cognitiva. Este fato sugere que a interferência produzida por imagens é mais intensa do que a produzida por palavras, sendo recomendada a primeira para avaliação da interferência em amostras de participantes sem histórico de trauma.

No entanto, o padrão de correlações desses fatores com traços de personalidade e raciocínio abstrato, mostra independência da medida desse novo 
instrumento desses construtos. Assim, seria interessante a realização de novas pesquisas para saber se os fatores obtidos nesta pesquisa podem ser associados à regulação de emoções propriamente dita ou não. A inexistência de correlação entre o fator N2 (Instabilidade Emocional) da Bateria Fatorial de Personalidade (BFP) com os fatores do instrumento alvo deste estudo sugere que não, pois pontuações elevadas em N2 descrevem características opostas à regulação emocional (irritação, nervosismo, oscilações de humor sem motivo aparente, impulsividade, tomadas de decisões precipitadas, dificuldade para controlar sentimentos negativos e baixa tolerância à frustração) (Nunes, Hutz \& Nunes, 2010), pelo que se esperava a obtenção uma correlação negativa e significativa com esse fator. Porém, a BFP é um instrumento destinado à avaliação dos cinco grandes fatores em população normal, ou seja, ela capta tendências, mas não é um instrumento sensível transtornos. Isto, aliado ao fato de que não houve seleção de participantes com algum tipo de transtorno de personalidade ou humor, pode ter reduzido a variabilidade das pontuações no teste alvo deste estudo, diminuindo as chances de encontrar correlações estatisticamente significativas. Assim, sugere-se que, em estudos posteriores sejam incluídas amostras clínicas, para aumentar a variabilidade das respostas às tarefas propostas no TRE.

Outra hipótese explicativa aos resultados obtidos neste estudo se refere ao número de estímulos (15) utilizados em cada bloco na presente versão do TRE. Esse número parece ter sido baixo, pois gerou diferenças entre os tempos de reação que, em sua maioria, giraram em torno de 2 segundos. Considerando que a cronometragem foi realizada manualmente, o erro ocorrido no disparo e na interrupção da cronometragem, pode ter significado uma boa porcentagem da diferença encontrada. Ou seja, a diferença encontrada pode conter considerável variância de erro e não de variância verdadeira no construto avaliado. Assim, sugere-se que em pesquisas futuras, o número de estímulos seja aumentado, e, se possível, que a cronometragem seja automatizada.

Apesar dessas limitações, a presente pesquisa apresenta uma possibilidade de mensuração da regulação de emoções, por meio de uma tarefa de desempenho. Alguns caminhos para a continuidade do desenvolvimento do instrumento foram apontados, abrindo novos horizontes para a realização de pesquisas futuras, com vistas à obtenção de um instrumento válido e fidedigno para avaliação da capacidade de regular emoções. 


\section{REFERÊNCIAS}

Ashley, V., \& Swick, D. (2009). Consequences of emotional stimuli: age differences on pure and mixed blocks of the emotional Stroop. Behavioral and Brain Functions, 5, 14, 1-11.

Bastian, V. A., Burns, N. R., \& Nettelbeck, T. (2005). Emotional intelligence predicts life skills, but not as well as personality and cognitive abilities, Personality and Individual Differences, 39, 1135-1145.

Bradley, M. M., \& Lang, P. J. (2007). The International Affective Picture System (IAPS) in the study of emotion and attention. In J. A. Coan and J. J. B. Allen (Eds.), Handbook of Emotion Elicitation and Assessment (pp. 29-46). Oxford University Press.

Bueno, J. M. H. (2008). Construção de um instrumento para avaliação da inteligência emocional em crianças. Tese de doutorado, Programa de PósGraduação Stricto Sensu em Psicologia, Universidade São Francisco, Itatiba.

Caruso, D. R., Mayer, J. D., \& Salovey, P. (2002). Relation of an Ability Measure of Emotional Intelligence to Personality. Journal of Personality Assessment, 79, 306-320.

Cisler, J. M., Wolitzky-Taylor, K. B., Adams Jr., T. G., Babson, K. A., Badour, C. L., \& Willems, J. L. (2011). The emotional Stroop task and posttraumatic stress disorder: A meta-analysis. Clinical Psychology Review, 31, 817-828.

Cobêro, C. (2004). Inteligência emocional: Validade do MSCEIT no contexto organizacional. Dissertação de Mestrado, Universidade São Francisco. Itatiba

Cobêro, C., Primi, R., \& Muniz, M. (2006). Inteligência emocional e desempenho no trabalho: Um estudo com MSCEIT, BPR-5 e 16PF. Paidéia, 16(35), 337487.

Dantas, M. A. (2004). Evidências de validade do Mayer Salovey Caruso Emotional Intelligence Test (MSCEIT). Dissertação de Mestrado, Universidade São Francisco, Itatiba.

Fava, D. C., Kristensen, C. H., Melo, W. V., \& Araujo, L. B (2009). Construção e validação de tarefa de Stroop Emocional para avaliação de viés de atenção em mulheres com Transtorno de Ansiedade Generalizada. Paideia, 19, 159165. 
Freitas, F. A., \& Noronha, A. P. P. (2006). Inteligência emocional e avaliação de alunos e supervisores: Evidências de validade. Psicologia: Teoria e Prática, $8(1), 77-93$.

Gotlib, I. H., \& McCann, C. D. (1984). Construct accessibility and depression: An examination of cognitive and affective factors. Journal of Personality and Social Psychology, 47, 427-439.

Jesus Jr., A. G. (2004). Estudo de validade e precisão do Mayer - Salovey Caruso Emotional Intelligence Test. Dissertação de Mestrado, Universidade São Francisco, Itatiba.

Kristensen, C. H., Gomes, C. F. de A., Justo, A. R., \& Vieira, K. (2011). Normas brasileiras para o Affective Norms for English Words. Trends in Psychiatry and Psychotherapy, 33(3), 135-146. Acessado em 15 de outubro de 2013 em http://www.scielo.br/scielo.php?script=sci_arttext\&pid=S2237$60892011000300003 \&$ Ing $=$ en\&tlng $=$ pt.

$10.1590 / S 2237-$ 60892011000300003.

Lang, P. J., Bradley, M. M., \& Cuthbert, B. N. (2005). International Affective Picture System (IAPS): Affective ratings of pictures and instruction manual. Technical Report A-6. Gainesville: University of Florida. http://www.unifesp.br/dpsicobio/adap/instructions.pdf. Acessado em out/2013.

Lasaitis, C., Ribeiro, R. L., Freire, M. V., \& Bueno, O. F. A. (2008). Atualização das normas brasileiras para o International Affective Picture System (IAPS), Revista de Psiquiatria do Rio Grande do Sul, 30(3), 230-235.

Mayer, J. D., \& Salovey, P. (1997). What is emotional intelligence? In: P. Salovey \& D. J. Sluyter (Eds.). Emotional Development and Emotional Intelligence: Implications for Educators, (pp. 3-31), New York, Basic Books.

Mayer, J. D., Salovey, P., \& Caruso, D. R. (2000). Emotional Intelligence as zeitgeist, as personality, and as mental ability. In: Reuven Bar-On \& James D. A. Parker (Orgs.), The handbook of emotional intelligence: theory development, assessment, and applications at home, school, and in the workplace. San Francisco: Jossey-Bass Inc, p. 92-117.

Mayer, J. D., Salovey, P., \& Caruso, D. R. (2002). Mayer-Salovey-Caruso Emotional Intelligence Test (MSCEIT) User's Manual. Toronto, Canada: MHS Publishers. 
Mayer, J. D., Salovey, P., Caruso, D. R., \& Sitarenios, G. (2003). Measuring Emotional Intelligence with the MSCEIT V2.0. Emotion, 3, 97-105.

Mayer, J. D., Caruso, D., \& Salovey, P. (1999). Emotional Intelligence meets standards for an intelligence. Intelligence, 27, 267-298.

Nunes, C. H., Hutz, C., \& Nunes, M. F. O. (2010). Bateria Fatorial de Personalidade. São Paulo, Casa do Psicólogo.

Palmer, B. R., Gignac, G., Manocha, R., \& Stough, C. (2005). A psychometric evaluation of the Mayer-Salovey-Caruso Emotional Intelligence Test Version 2.0. Intelligence, 33, 285-305.

Pelletteri, J. (1999). The relationship between emotional intelligence, cognitive reasoning, and defense mechanisms. Dissertation Abstracts International: Section B: The Sciences \& Engineering, 60, 403.

Primi, R., \& Almeida, L. S. (2000). Baterias de Provas de Raciocínio (BPR-5): Manual técnico. São Paulo: Casa do Psicólogo.

Ribeiro, R. L., Pompéia, S., \& Bueno, O. F. A. (2004). Normas brasileiras para o International Affective Picture System (IAPS): Comunicação breve, Revista de Psiquiatria do Rio Grande do Sul, 26(2), 190-194.

Salovey, P., \& Mayer, J. D. (1990). Emotional Intelligence. Imagination, Cognition and Personality, 9, 185-211.

Smith, P., \& Waterman, M. (2004). Processing Bias for Sexual Material: The Emotional Stroop and Sexual Offenders. Sexual Abuse: A Journal of Research and Treatment, 16, 163-171.

Zeidner, M., Matthews, G., \& Roberts, R. D. (2001). Slow down, you move fast: Emotional Intelligence remains an 'elusive' intelligence. Emotion, 1, 265275.

Contato: mauricio.ufpe@gmail.com

Recebido em: 18/01/2014

Revisado em: 01/03/2014

Aceito em: 08/03/2014 